\title{
Abdominal Pregnancy Implanted in the Spleen: A Case Report
}

\author{
*Md. Nure Alom Siddiqui ${ }^{1}$, Md. Tohurul Islam ${ }^{2}$, Fatema Siddiqua ${ }^{3}$ \\ Shahnaj Sultana ${ }^{4}$, Abu Bakar Siddique ${ }^{5}$ \\ ${ }^{1}$ Dr. Md. Nure Alom Siddiqui, Jr. Consultant (Medicine) Rajshahi Medical College \\ ${ }^{2}$ Dr. Md. Tohurul Islam ${ }^{2}$ Asstt. Professor of Radiology \& Imaging, SZMC, Bogra \\ ${ }^{3}$ Dr. Fatema Siddiqua, Asst. Prof of Gynae \& Obstetrics IBMC, Rajshahi \\ ${ }^{4}$ Dr. Shahnaj Sultana, MO, UHC Paba, Rajshahi \\ ${ }^{5}$ Dr. Abu Bakar Siddique, Senior Consultant, Nator Sadar Hospital \\ *Corresponding Author
}

\begin{abstract}
Primary splenic pregnancy is the least common form of extrauterine pregnancy. We report a case of splenic pregnancy occurring in a 30-year-old woman presenting with acute abdomen and hemoperitoneum. Recognition of this rare form of gestation is of critical importance, owing to the risk of exsanguinations and death, and should be considered in the differential diagnosis of acute abdomen in women of reproductive age. We report a case of primary splenic pregnancy complicated by hemoperitoneum.
\end{abstract}

Key Words : Ectopic Splenic Pregnancy, Acute Abdomen, Reproductive Age

\section{Introduction}

Ectopic pregnancy is deined as implantation of a fertilized ovum anywhere other than within the uterine cavity and occurs with an estimated incidence of 19.7 per 1000 pregnancies $^{1}$ The most $^{-1}$ common site of ectopic implantation is within the fallopian tube, accounting for $95.5 \%$ of all ectopic gestations. ${ }^{2}$ Although rare, extratubal pregnancies represent some of the most serious complications of pregnancy. Approximately $1.3 \%$ of ectopic pregnancies are abdominal ${ }^{2}$ and occur with direct implantation onto the peritoneal surface. Primary abdominal pregnancies have been described in a variety of extrapelvic organs, including omentum, liver, and small and large intestine. Preoperative diagnosis of abdominal pregnancy is difficult and patients commonly present with signs of shock and hemoperitoneum. The spleen is one of the rarest sites for ectopic gestation and to our knowledge, only 9 cases of primary splenic pregnancy have been documented in the English literature to date ${ }^{3-11}$.

\section{Report of a case}

A 30-year-old, gravida 3, para 2, woman status post uncomplicated normal vaginal delivery 7 years earlier, presented with severe left upper quadrant pain. The patient reported abdominal "fullness" that began several weeks before presentation. On the morning of presentation, the discomfort progressed to severe left upper quadrant pain aggravated by movement and deep breathing. The pain radiated to the left shoulder with deep inspiration. The patient also reported feeling lightheaded and dizzy. At the time of admission, she was amenorrhoeic for last three months. A urine $\beta$-human chorionic gonadotropin (Pregnancy) test was positive. Physical examination revealed a patient in obvious distress with moderately anaemic. She was afebrile but diaphoretic and hypotensive, with a blood pressure of 90/60 mm Hg. Her abdominal examination was significant for diffuse abdominal tenderness with rebound and guarding. Bimanual examination was not performed. 


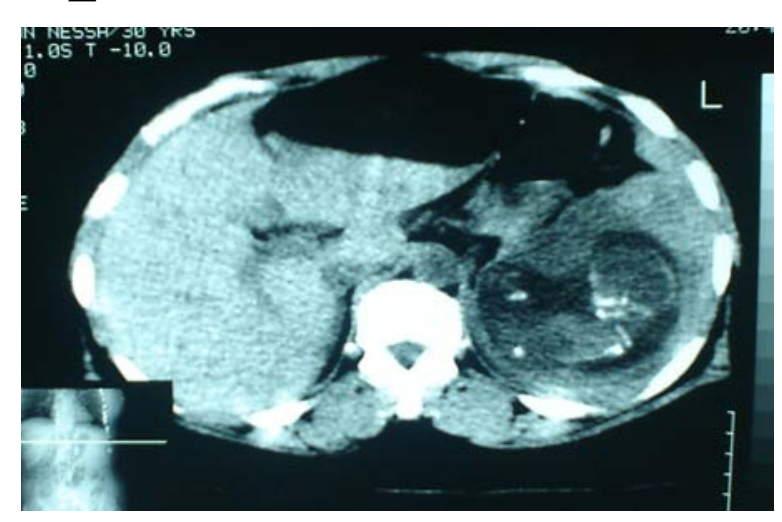

Fig. 1: CT scan of abdomen shows fetus attached the spleen

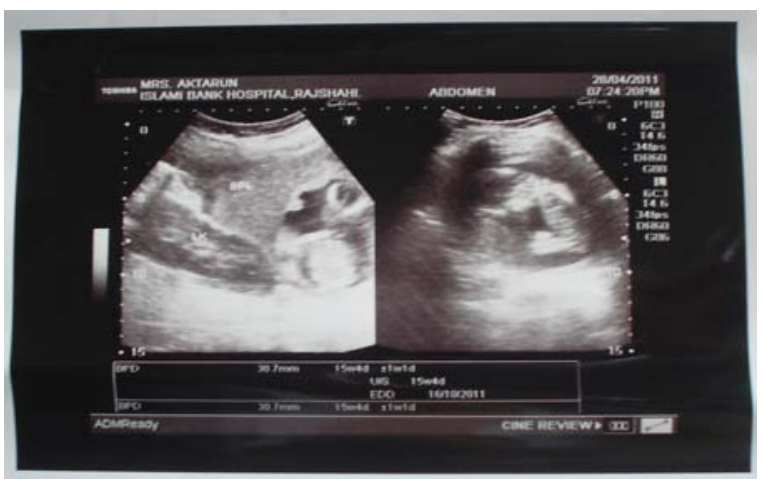

Fig. 2: USG Abd .shows Fetus in the spelinic region

An abdominal ultrasound revealed a fetus attached with spleen with moderate peritoneal collection. CT scan of abdomen showed foetus was attached with spleen. The patient was taken for emergency laparotomy for suspected splenic rupture with hemoperitoneum. Intraoperatively, $1.5 \mathrm{~L}$ of blood and blood clot were found within the peritoneal cavity. The placenta of fetus was attached with the spleen. Further inspection of the pelvic cavity revealed no abnormality. No tubal pregnancy was identified. No other source of bleeding was noted. Fetus was removed from spleen with moderate bleeding. The patient recovered uneventfully.

\section{Discussion}

Abdominal pregnancy is an extremely rare event that may be difficult to diagnose. The advanced gestational age at which most abdominal pregnancies are discovered complicates management further.
Md. Nure Alom Siddiqui, Md. Tohurul Islam, Fatema Siddiqua et al

Because of the propensity for hemorrhage, removal of abdominal pregnancies requires surgical extraction and discrimination in deciding if placental removal is prudent. There is little information known about future fertility after abdominal pregnancy.

Abdominal pregnancies are defined as those occurring within the peritoneal cavity (excluding tubal, ovarian, or intraligamentous pregnancies) and account for $1.3 \%$ of ectopic pregnancies ${ }^{2}$. The incidence of abdominal pregnancy is estimated at 1 in 8000 births $^{12}$. Such pregnancies are potentially life-threatening, with a maternal mortality rate of 5.1 per 1000 cases, which is 7.7 times higher than the risk from other ectopic pregnancies and approximately 90 times higher than that associated with intrauterine pregnancy ${ }^{13}$. The risk is particularly critical without an accurate preoperative diagnosis. Abdominal pregnancies are classified as either primary or secondary; the latter, which is much more common, is associated with tubal rupture followed by implantation at a second site (eg, the peritoneal surface). Primary abdominal pregnancies, which arise from fertilization of an ovum within the peritoneal cavity, are extremely rare. According to Studdiford, ${ }^{14}$ the criteria for primary abdominal pregnancy are as follows: (1) fallopian tubes and ovaries are grossly normal and show no evidence of recent injury; (2) no evidence of uteroplacental fistula; and (3) a pregnancy of no more than 12 weeks' gestation with trophoblastic elements related exclusively to a peritoneal surface. The third criterion ensures that the pregnancy is immature enough to exclude the possibility of secondary implantation to the peritoneal cavity after primary tubal pregnancy rupture. In our case, focal adhesions were noted during intraoperative examination of the fallopian tubes, but no other abnormalities were idefined. There was no evidence of pregnancy outside the spleen, therefore satisfying the criteria established for primary splenic pregnancy.

Although the most common sites of primary peritoneal pregnancy are the pouch of Douglas and the posterior uterine wall, ${ }^{13}$ primary implantation sites have been described in extrapelvic structures, including small and large intestine, ${ }^{13}$ omentum, ${ }^{11}$ liver, ${ }^{11}$ and spleen ${ }^{1-11}$. Risk factors associated with abdominal pregnancies are similar to those of other ectopic pregnancies and include prior history 
Abdominal Pregnancy Implanted in the Spleen: A Case Report

of pelvic inflammatory disease, ectopic gestation, endometriosis, infertility with subsequent in vitro fertilization, and previous tubal surgery ${ }^{15}$. Our review of previously published reports of primary splenic pregnancies revealed a mean age of 27.3 years (range, 23-37 years). As with our case, most patients presented with sudden-onset left upper quadrant abdominal pain that radiated to the left shoulder. 8 of the 9 previously published cases had preoperative diagnoses of ruptured ectopic pregnancy, with the remaining case undiagnosed until the results of histologic examination. All patients survived. A variety of splenic implantation sites have been reported and range from superior to lower pole and hilum. Most gestations manifested as capsular projections and all were sub capsular in location. Atrash et al ${ }^{13}$ showed that abdominal pregnancy-associated deaths occur later in gestation compared to other ectopic pregnancies. Primary splenic pregnancy tends to present earlier than other abdominal pregnancies, presenting with hemoperitoneum occurring at 6 to 8 weeks'gestation. Interestingly, in 9 of 10 splenic gestations the size ranged from 2.0 to $3.5 \mathrm{~cm}$, suggesting that rupture of the splenic capsule occurs when the ectopic gestation exceeds this size. Three of the 10 patients described had a known risk factor for ectopic pregnancy, namely, the presence of an intrauterine contraceptive device. There were no fetal parts identified in any of the reported cases. The incidence of ectopic pregnancy in the United States has been steadily increasing. Atrash et $\mathrm{al}^{13}$ found that 3 to 4 women a year in the United States die of abdominal pregnancy. In this study, Atrash et $\mathrm{al}^{13}$ found that only 1 of 9 women alive at hospital admission had an accurate preoperative diagnosis of abdominal pregnancy and suggested that preventing abdominal pregnancy-related death depends in part on increasing awareness of its clinical characteristics. Although recognition of primary abdominal pregnancy poses a difficult diagnostic challenge, increased detection of ectopic pregnancies is made possible through improved access to ultrasonography and improved sensitivity of urine and serum $\beta$-human chorionic gonadotropin tests.

\section{Conclusion}

Recognition of this rare form of gestation is of critical importance owing to the risk of exsanguination and death, and should be considered in the differential diagnosis of acute abdomen in women of reproductive age.

\section{References}

1. Center for Disease Control and Prevention. Ectopic pregnancy: United States, 1990-1992. JAMA. 1995; 273: 533

2. Bouyer J, Coste J, Fernandez H, Pouly JL, Job-Spira N. Sites of ectopic pregnancy: a 10-year population-based study of 1800 cases. Hum Reprod. 2002; 17: 3224-3230

3. Mankodi RC, Sankari K, Bhatt SM. Primary splenic pregnancy. Br J Obstet Gynaecol. 1977; 84: 634-635

4. Reddy KSP, Modgill VK. Intraperitoneal bleeding due to primary splenic pregnancy. Br J Surg. 1983; 70: 564

5. Huber DE, Martin SD, Orlay G. A case report of splenic pregnancy. Aust N Z J Surg. 1984; 54: 81-82

6. Caruso V, Hall WH. Primary abdominal pregnancy in the spleen: a case report. Pathology. 1984; 16: 93-94

7. Tantachamroon T, Songkrobhan S, Tuppasut NK. Primary splenic pregnancy. J Med Assoc Thai. 1986; 69: 495-499

8. Larkin JK, Garcia DM, Paulson EL, Powers DW. Primary splenic pregnancy with intraperitoneal bleeding and shock: a case report. Iowa Med. 1988; 78: 529-530

9. Yackel DB, Panton ON, Martin DJ, Lee D. Splenic pregnancy: case report. Obstet Gynecol. 1988; 71: 471473

10. Kahn JA, Skjeldestad FE, v During V, Sunde A, Molne $\mathrm{K}$, Jorgensen OG. Aspleen pregnancy. Acta Obstet Gynecol Scand. 1989; 68: 83-84

11. Cormio G, Santamato S, Vimercati A, Selvaggi L. Primary splenic pregnancy: a case report. J Reprod Med. 2003; 48: 479-481

12. Rojansky N, Schenjer JG. Heterotopic pregnancy and assisted reproduction: an update. J Assist Reprod Genet. 1996; 13: 594-601

13. Atrash HK, Friede A, Hogue C. Abdominal pregnancy in the United States: frequency and maternal mortality. Obstet Gynecol. 1987; 69: 333-337

14. Studdiford WE. Primary peritoneal pregnancy. Am $J$ Obstet Gynecol. 1942; 44: 487-491

15. Strafford JC, Ragan WD. Abdominal pregnancy: review of current management. Obstet Gynecol. 1977; 50: 548 